Equation (5) is an immediate consequence of (4), and the inequality (6) follows from (5) and (2). The first statement of the theorem is thus verified and the second statement becomes evident when $\Pi(t+\Delta t)$ is expanded in a Taylor's series in $\Delta t$ about the time $t$.

If the complementary energy is defined by

$$
\Pi_{c} \equiv \int_{V}\left(\int \epsilon_{i j} d \sigma_{i j}\right) d V-\int_{S_{D}} T_{i} u_{i}
$$

and a functional $W_{c}$ by

$$
W_{c}\left(\sigma_{0}, \epsilon_{*}\right)=\int_{V} \sigma_{i j}^{0} \epsilon_{i j}^{*}-\int_{S_{D}} T_{i}^{0} u_{i}^{*}
$$

then the elastic principle of complementary energy states that $\Pi_{c}$ is a minimum for the actual state among all statically admissible states and the analogous plastic principle states that

$$
W_{c}\left(\sigma_{0}^{\prime}, \epsilon_{0}^{\prime}\right)-W_{c}\left(\sigma^{\prime}, \epsilon^{\prime}\right) \geq 0 .
$$

Just as for the first principle one can easily prove two consequences of (9).

Theorem. Among all statically admissible rate states the actual rate state minimizes the time variations of

(1) the complementary dissipation function $W_{c}\left(\sigma^{\prime}, \epsilon\right)$ :

(2) the complementary energy $\Pi_{c}$.

\title{
ReFERENCES
}

1. R. Hill, The mathematical theory of plasticity, Oxford Univ. Press, London, 1950

2. W. Prager and P. G. Hodge, Jr., Theory of perfectly plastic solids, John Wiley and Sons, Inc., New York, 1951

3. J. N. Goodier and P. G. Hodge, Jr, Elasticity-plasticity, John Wiley and Sons, Inc., New York, 1958

4. D. C. Drucker, Variational principles in the mathematical theory of plasticity, Proceedings of Symposium on Calculus of Variations, (Chicago, 1956), McGraw-Hill Book Co., Inc., New York, 1958, pp. 3-22.

5. W. T. Koiter, General theorems for elastic-plastic solids, Progress in solid mechanics, vol. 1, Chap. 4, North-Holland Publ. Co., Amsterdam, 1960

\section{ON THE SIMULTANEOUS DIAGONALIZATION OF TWO SEMI-DEFINITE MATRICES*}

\section{BY ROBERT W. NEWCOMB (University of California, Berkeley)}

1. Introduction. The use of congruency transformations for simultaneously diagonalizing two symmetric matrices, one of which is definite, is well known. One merely diagonalizes the definite matrix to (plus or minus) unity. This is then followed by an orthogonal transformation which diagonalizes the other matrix while preserving the unit matrix already obtained [1]. If, instead of being definite, one matrix is semi-definite,

*Received July 11, 1960. 
this method fails. However, if both matrices are semi-definite, this standard procedure can be extended.

2. Diagonalization. In the following let the superscript $t$ denote matrix transposition and $1_{r}$ denote the unit matrix of order $r$. Further let the $r$ th order zero matrix be denoted by $0_{r}$. The main result is then the following theorem.

Theorem: Let $A$ and $B$ be $n x n$ real, symmetric, positive semi-definite matrices. Then there exists a real non-singular matrix $T$ and real diagonal matrices $A_{0}$ and $B_{0}$, [see Eqs. (3) \& (8)], such that

$$
\begin{aligned}
& A=T^{t} A_{o} T \\
& B=T^{t} B_{o} T
\end{aligned}
$$

Proof: Let $A$ have rank $a$ and $B$ rank $b$ and assume that $b \geq a$. We first find a real, non-singular $T_{0}$ such that

$$
\begin{aligned}
& A=T^{t} A_{\circ} T_{\circ} \\
& B=T^{t}{ }_{0} B^{\prime} T_{\circ}
\end{aligned}
$$

where

$$
A_{\circ}=\text { diagonal }\left[1_{a}, 0_{n-a}\right] .
$$

If any of the last $n-a$ diagonal elements of $B^{\prime}$ are zero, the corresponding entire row and column of $B^{\prime}$ are zero, since $B^{\prime}$ is semi-definite. For the last $n-a$ diagonal elements of $B^{\prime}$ which are nonzero, we can reduce the remaining nondiagonal elements in these rows and columns to zero. We must do this by always adding the diagonal element to the off diagonal element in order to preserve $A_{0}$. We can then write

$$
\begin{aligned}
& A=T_{o}^{t} T_{1}^{t} A_{o} T_{1} T_{。} \\
& B=T_{o}^{t} T_{1}^{t} B^{\prime \prime} T_{1} T_{。}
\end{aligned}
$$

where

$$
B^{\prime \prime}=\left[\begin{array}{c:cc}
B_{a} & & 0 \\
\hdashline & 1_{b-\beta} & \\
0 & & 0_{n-a-b+\beta}
\end{array}\right]
$$

Here $\beta \geq 0$ is defined as the rank of $B_{a}$. We now diagonalize $B_{a}$ by an orthogonal transformation $T_{a}$ and put

$$
T_{2}=\left[\begin{array}{c:c}
T_{a} & 0 \\
\hdashline 0 & 1_{n-a}
\end{array}\right]
$$

Now let

$$
T=T_{2} T_{1} T_{\circ}
$$

Then Eq. (1) results with

$$
B_{o}=\operatorname{diagonal}\left[\lambda_{1}, \cdots, \lambda_{\beta}, 0_{a-\beta}, 1_{b-\beta}, 0_{n-a-b+\beta}\right]
$$

where $\lambda_{i}>0, i=1, \cdots, \beta$. 
By observing that neither the "sign" of $A_{0}$ nor that of $B_{0}$ enters into the proof, we see that we can diagonalize two semi-definite matrices (possibly of opposite sign). We can also easily extend the theorem to Hermitian matrices. Thus let a superscript asterisk denote complex conjugation and let $A$ and $B$ be complex Hermitian, positive semidefinite matrices. Proceeding as above, but using complex $T_{0}, T_{1}$ and unitary $T_{a}$, we can write

$$
\begin{aligned}
& A=T^{t^{*}} A_{o} T \\
& B=T^{t^{*}} B_{o} T
\end{aligned}
$$

where $A_{0}$ and $B_{0}$ are as in Eqs. (3) and (8).

3. Applications. The above theorem is necessary for the synthesis of networks which are passive or active at a point (to be published, for the basic concepts see [2]). It also can be used to advantage in the synthesis of two element kind networks, as well as in studying equivalent networks (see pp. 96 and 142 of [3]). Its use in studying the vibraticns of systems satisfying Lagrange's equations should also be apparent.

\section{REFERENCES}

1. H. Turnbull and A. Aitken, "An Introduction to the Theory of Canonical Matrices," Blackie \& Son 1952 , p. 107.

2. C. Desoer and E. Kuh, "Bounds on Natural Frequencies of Linear Active Networks," Proceedings of the Brooklyn Polytechnic Symposium on Active Networks and Feedback Systems, (1960).

3. E. A. Guillemin, "Synthesis of Passive Networks," John Wiley \& Sons, Inc., New York, 1957.

\section{AN UPPER BOUND ON RIGHT HALF PLANE ZEROS*}

\section{By DOV HAZONY (Case Institute of Technology)}

Abstract. An upper bound is placed on the number of right half plane zeros of functions of the type $Z-m / n . Z$ and $m / n$ are RLC and LC driving point impedance functions respectively. In addition, it is shown that if $\operatorname{Re} Z>0$ on $j$ axis, the number of right half plane zeros is determined precisely.

Introduction. In problems of control and network synthesis, it may be necessary to determine the number of right half plane zeros of certain impedance functions. In control problems, zeros in the right half plane may cause instability while in synthesis they may require active networks. In this paper an upper bound is placed on the number of these zeros of the class of functions $Z-m / n$ and $Z-n / m$. These terms are defined below.

\section{Lemma.}

Given: I. $\quad Z$ is prf (an RLC driving point impedance function).

II. $m+n$ is a Hurwitz polynomial, of degree $d$, of the complex variable $S ; m$ is an even and $n$ is an odd function of $S$.

${ }^{*}$ Received July 27, 1960. This paper, although based on the work sponsored by the U. S. Air Force, Cambridge Research Center, Bedford, Mass. Contract No. AF 19(604)3887, has not been approved or disapproved by that agency. 